\title{
SM AND BSM HIGGS RESULTS FROM THE CMS EXPERIMENT*
}

\author{
ARTUR Kalinowski \\ on behalf of the CMS Collaboration
}

Faculty of Physics, University of Warsaw, Hoża 69, 00-681 Warszawa, Poland

(Received April 14, 2014)

Results of CMS Collaboration Higgs boson searches based on the Run 1 LHC data are presented. Details of searches in various Standard Model Higgs decay modes are presented. Results of searches for MSSM Higgs bosons in $\tau \tau$ and $b \bar{b}$ channels are presented as well.

DOI:10.5506/APhysPolB.45.1415

PACS numbers: 14.80.Da, 14.80.Bn

\section{Introduction}

Until July $4^{\text {th }}, 2012$ the Higgs boson was the last missing piece of the Standard Model. Observation of a new resonance in the $\gamma \gamma$ and $Z Z$ proclaimed by the ATLAS [1] and CMS [2] collaborations [3, 4] opened a new era in high energy physics. The initial observation, based on about $5 \mathrm{fb}^{-1}$ of data collected at collision energy of $7 \mathrm{TeV}$, was only enough to establish existence on a new boson with mass of about $125 \mathrm{GeV}$. The final identification of the new state as a Higgs boson requires observation in all available decay and production modes, and detailed comparison of measured properties, like couplings and quantum numbers with Standard Model (SM) or beyond SM (BSM) expectations. Such a program was continued after July $4^{\text {th }} 2012$, until end of the so-called LHC Run 1 in February 2013, and will be continued after the LHC restart in 2015.

In this contribution, we briefly describe the results of searches in the main SM decay and production modes. Since the BSM spans a large number of variants, we present only the most expected one, that is a result of searches for the MSSM Higgs bosons.

\footnotetext{
* Presented at the Cracow Epiphany Conference on the Physics at the LHC, Kraków,
} Poland, January 8-10, 2014. 


\section{SM production and decay modes}

The main modes for the Standard Model Higgs production at the protonproton collider are gluon-gluon fusion, yielding cross sections of the order of $20 \mathrm{pb}$ for Higgs mass $125 \mathrm{GeV}$ at the NNLO+NNLL QCD+NLO EW calculation order [5], see Fig. 1 (left). This channel, however, is very difficult from the experimental point of view, as it does not provide any additional handle, besides Higgs decay products, for signal selection from overwhelming QCD background. This is particularly difficult for fully hadronic Higgs decay final state searches, like the $H \rightarrow b \bar{b}$ mode. The next production mode, yielding cross section of the order of $1.6 \mathrm{pb}$ is a so-called vector boson fusion (VBF), where the Higgs boson is emitted from vector bosons, which, in turn, are emitted from incoming quarks, being thus scattered into the final state. The jets originating from hadronization of scattered quarks provide a very good handle to select the signal. The two following production modes are associated production with $W$ or $Z$ bosons, with cross section of the order of $0.5 \mathrm{pb}$, and $t \bar{t}$ associated production, with cross section as low as $0.13 \mathrm{pb}$, but with excellent signatures from decays of associated particles. All above production modes are exploited by the CMS Collaboration, which allows to maximize the potential and robustness of Higgs boson related analyses. Most of the analyses define event categories enhancing specific production modes, in particular the VBF one.
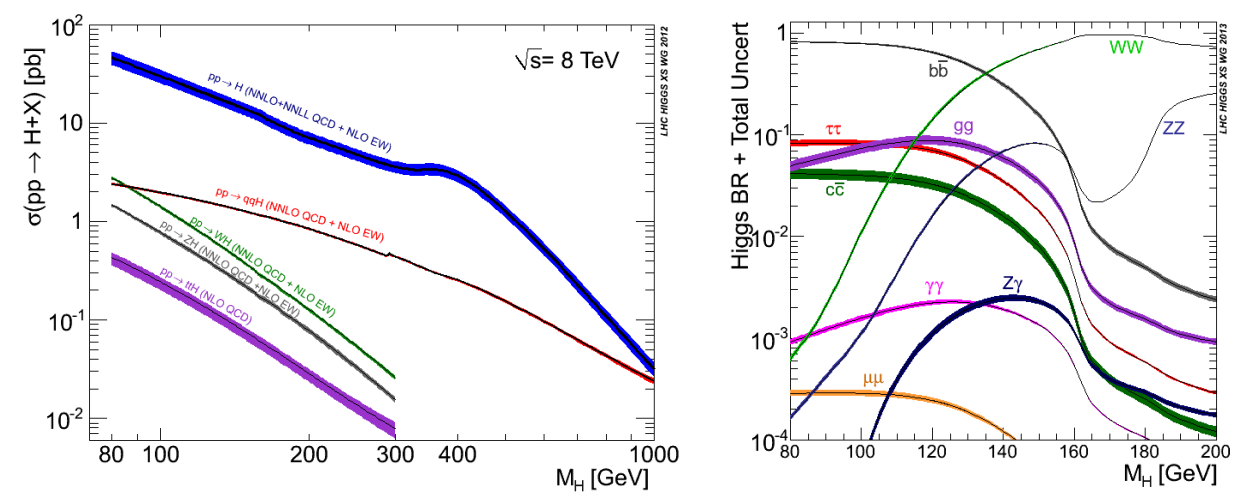

Fig. 1. Standard Model Higgs boson production cross sections for $8 \mathrm{TeV} p p$ collisions (left), and decay branching ratios (right) [5].

The Higgs boson of mass around $125 \mathrm{GeV}$ has a very rich decay pattern, as shown in Fig. 1 (right). At this mass, there are many decay modes with relatively large branching fractions [5]. All decay modes accessible at the hadron collider were analyzed by the CMS Collaboration, the only ones remaining are gluon-gluon and light-quark decay modes, which seem to be completely inaccessible at the LHC. 


\section{The $\tau \tau$ coupling}

The search in the $\tau \tau$ decay mode [6] uses all six possible configurations of the $\tau$ leptons pair decays: $\mu \tau_{\mathrm{h}}, e \tau_{\mathrm{h}}, \tau_{\mathrm{h}} \tau_{\mathrm{h}}, e \mu, \mu \mu$ and $e e$ where $\tau_{\mathrm{h}}$ denotes hadronic $\tau$ decays. The events are divided into number of categories following the production modes final states: 0 -jet category contains mostly events produced in $g g \rightarrow H$ process, 1-jet category aims at $g g H+j$, or VBF category, where one of jets is lost, and finally 2-jet category for the VBF process. To further enhance the sensitivity some categories are split according to $\tau_{\mathrm{h}}$ transverse momenta. The most sensitive category, the 2 -jet one, is further split into parts with loose and tight selection on the two tagging jets.

The invariant mass of the $\tau \tau$ pair cannot be reconstructed directly, as there are at least two neutrinos in the final state. The CMS Collaboration uses a likelihood approach to reconstruct the full mass with almost $100 \%$ efficiency with resolution of the order of $20 \%$, as can bee seen from Fig. 2 (left). The main background processes in this analysis come from irreducible $Z \rightarrow \tau \tau$ and reducible QCD dijets, and $W+$ jet processes. The main backgrounds are estimated from data using side bands for reducible backgrounds, and embedding of simulated events into real data for $Z \rightarrow \tau \tau$.
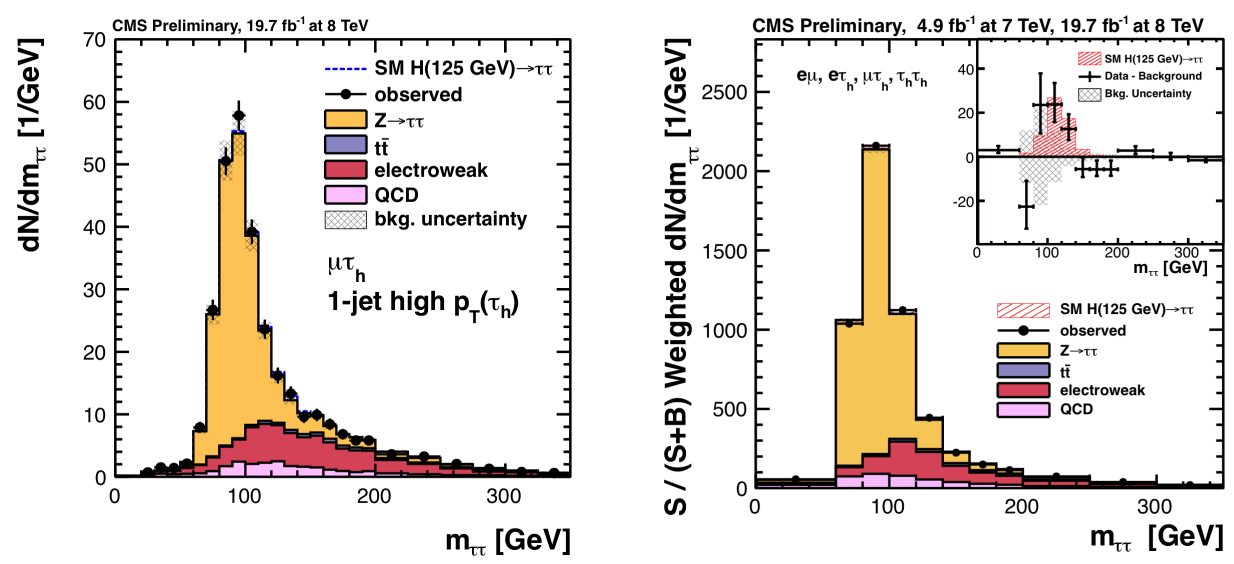

Fig. 2. Invariant mass of $\tau \tau$ system reconstructed with likelihood method approach for $\mu+\tau_{\text {jet }}$, one jet final state (left). The invariant mass distribution with each event weighted by the $\mathrm{S} /(\mathrm{S}+\mathrm{B})$ value of its category (right). The insert presents the background-subtracted distribution. Signal expectation and background estimates are shown.

After analyzing full dataset collected during LHC Run 1, a signal-like excess was observed around $m_{H}=125 \mathrm{GeV}$. The statistical significance of the excess corresponds to $3.4 \sigma$ which makes this an evidence of $H \rightarrow \tau \tau$. 
The best fit value for the mass is $m_{H}^{\text {best fit }}=115_{-2}^{+8} \mathrm{GeV}$, which is compatible with other mass measurements. Figure 2 (right) presents the invariant mass for all event categories, weighted by each category $\mathrm{S} /(\mathrm{S}+\mathrm{B})$ ratio.

\section{The $\mu \mu$ coupling}

The branching fraction of the SM Higgs to pair of muons is of the order of $2.2 \times 10^{4}$. This is even bigger than branching ratio for the "golden" $4 \mu$ channel, which has $\operatorname{BR}(H \rightarrow Z Z \rightarrow 4 \mu)=3.27 \times 10^{-5}$ at $m_{H}=125 \mathrm{GeV}$. The reason that $H \rightarrow \mu \mu$ is so difficult is the huge background from the DrellYan muon pair production. Nevertheless, the CMS Collaboration made an effort in looking for the signal in this channel too [7]. The $H \rightarrow \mu \mu$ analysis divides events into subcategories according to production mode, by requiring that events have zero, one or two jets, as well as muon pair $p_{\mathrm{T}}$. The signal is extracted by simultaneous fit of signal and background shapes to the observed mass spectrum. An example of reconstructed dimuon invariant mass with signal expectation multiplied by a factor 20 is presented in Fig. 3 (left). No signal excess was observed in the full Run 1 data sample, therefore an exclusion limit at 95\% C.L. was calculated. Figure 3 (right) presents the exclusion limit as a function of Higgs mass hypothesis. The observed exclusion limit on the so-called signal strength modifier $\sigma / \sigma_{\mathrm{SM}}$ is 7.4 for $m_{H}=125 \mathrm{GeV}$, while the expected one was 5.1. Much larger amounts of data are needed to allow exclusion or discovery of a signal in this channel. Exclusion limit could be reached with $175 \mathrm{fb}^{-1}$, and discovery with $450 \mathrm{fb}^{-1}$ of data collected at $\sqrt{s}=14 \mathrm{TeV}$.
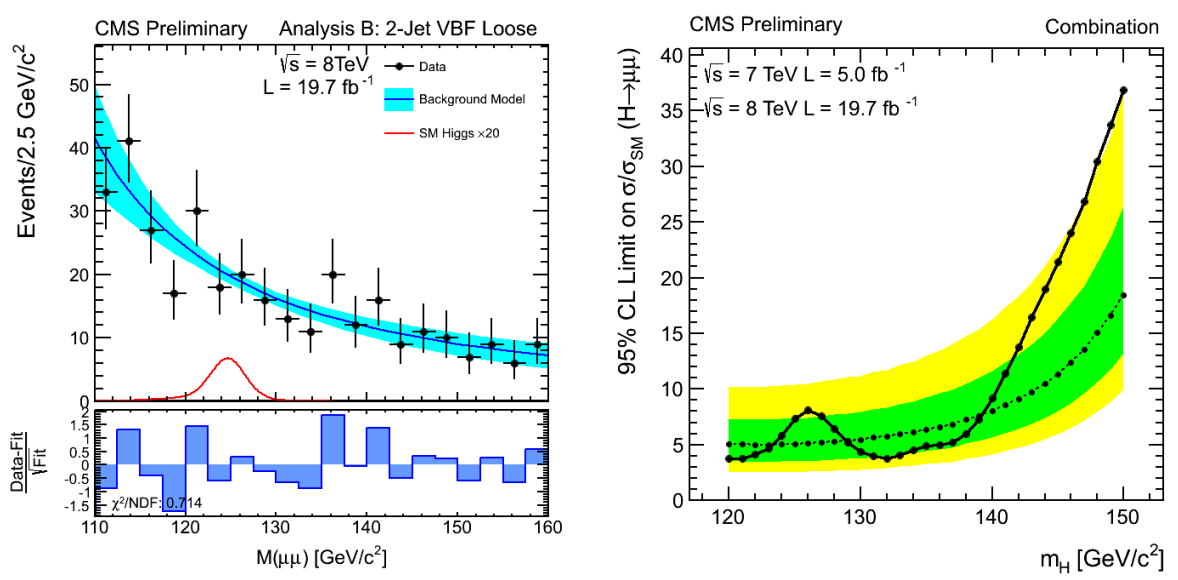

Fig. 3. Invariant mass of $\mu \mu$ pair in the VBF enhanced event category. Fitted background model and signal expectation multiplied by a factor 20 are overlaid (left). Exclusion limits for the $H \rightarrow \mu \mu$ search obtained with $5.0(19.7) \mathrm{fb}^{-1}$ of data at $7(8) \mathrm{TeV}$. 


\section{The $b \bar{b}$ coupling}

The $H \rightarrow b \bar{b}$ decay mode has largest value of the branching ratio, but being a fully hadronic final state is a very difficult. To allow efficient triggering and offline event selection it has to be combined with a production mode that has additional handles in the final state. The associated VH production mode is used, where leptonic $Z$ or $W$ decays provide necessary leptonic part [8]. The event selection in this analysis is made using Boosted Decision Tree (BDT) multivariate technique. The $\mathrm{BDT}$ is trained on $p_{\mathrm{T}}$ of the two $b$-jets, their invariant mass and $b$-tagging discriminator. The invariant mass reconstruction is improved by using a regression, which improves resolution by about 10\%, as presented in Fig. 4 (left). The signal is extracted by a simultaneous fit to the BDT discriminator spectrum in four categories enriched in $t \bar{t}, \mathrm{~V}+$ jets, dibosons backgrounds, and $\mathrm{VH}$ signal. The analysis found an excess of signal-like events, with statistical significance on the level of $2.1 \sigma$ at $m_{H}=125 \mathrm{GeV}$. Figure 4 (right) presents the $95 \%$ C.L. exclusion limit, where a broad excess of the observed limit with respect to background only expectation is clearly visible.
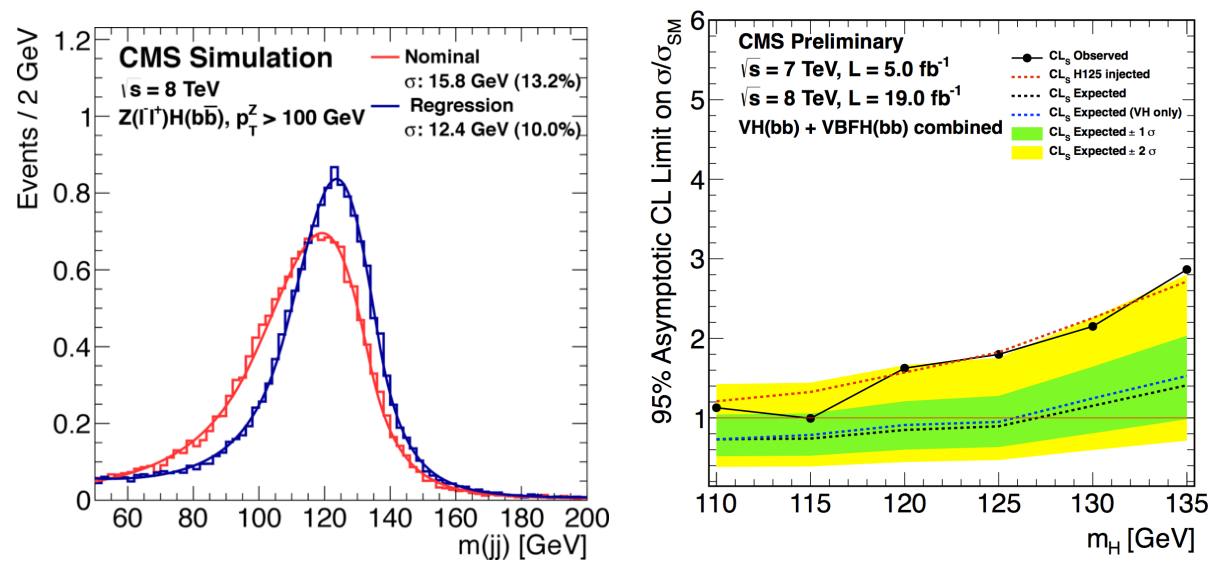

Fig. 4. Invariant mass of $b \bar{b}$ pair reconstructed from jet four momenta, and using the regression method (left). Exclusion limits for the $H \rightarrow b \bar{b}$ search obtained with $5.0(19) \mathrm{fb}^{-1}$ of data at $7(8) \mathrm{TeV}$.

The combination of two analyses looking for a Higgs decays to fermions: $H \rightarrow \tau \tau$ and $H \rightarrow b \bar{b}$ give $3.8 \sigma$ evidence for Higgs boson coupling to third generation fermions [9]. 


\section{The $t \bar{t}$ coupling}

The CMS Collaboration was looking for the associated production of the Higgs boson with $t \bar{t}$. The following Higgs decay channels were analysed: $W W / Z Z$ [10], $b \bar{b}$ [11, 12], $\gamma \gamma$ [13], and $\tau \tau$ [12]. Since the $t \bar{t} H$ cross section is very small, the results of the individual analyses are still laden with very large uncertainties, as can bee seen from Fig. 5, where the signal strength modifier found in each decay channel is shown. The combination of all decay modes shows a signal-like excess with statistical significance of $2.4_{-1.0}^{+1.1} \sigma$ at $m_{H}=125.7 \mathrm{GeV}$ showing an hint of Higgs boson coupling to top quarks.

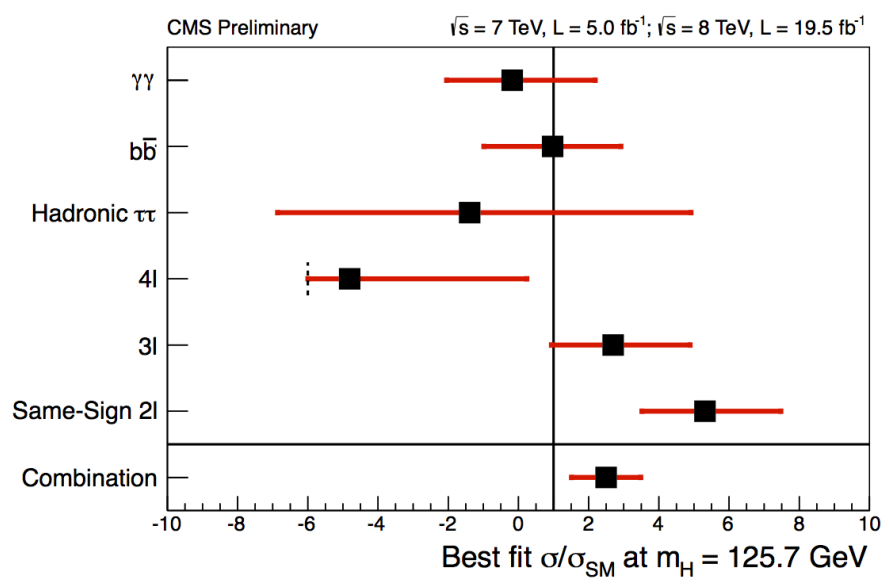

Fig. 5. Best fit values of signal strength modifier for the associated $t \bar{t} H$ production estimated in various Higgs decay modes obtained with $5.0(19.5) \mathrm{fb}^{-1}$ of data at 7(8) $\mathrm{TeV}$.

\section{The $V V$ couplings}

The search for $H \rightarrow W W \rightarrow l \nu l \nu$ has the best performance among all $W W$ decay modes. The analysis [15] looks for a broad peak in the transverse mass of leptons and missing transverse energy $\left(m_{\mathrm{T}}\right)$, since due to the presence of neutrinos in the final state, the full invariant mass cannot be reconstructed. The events are split into categories depending on the number of jets in the final state. The signal selection is separately optimized for a number of $m_{H}$ hypotheses, and the signal selection is based on $m_{H}$ shape analysis, or simple event counting, depending on the number of events in given event category. Figure 6 (left) presents the expected and observed signal significance as a function of $m_{H}$. Analysis of the full set of data collected in the LHC Run 1 shows a signal-like excess with statistical significance on the level of $4.3 \sigma$ at $m_{H}=125.6 \mathrm{GeV}$. 

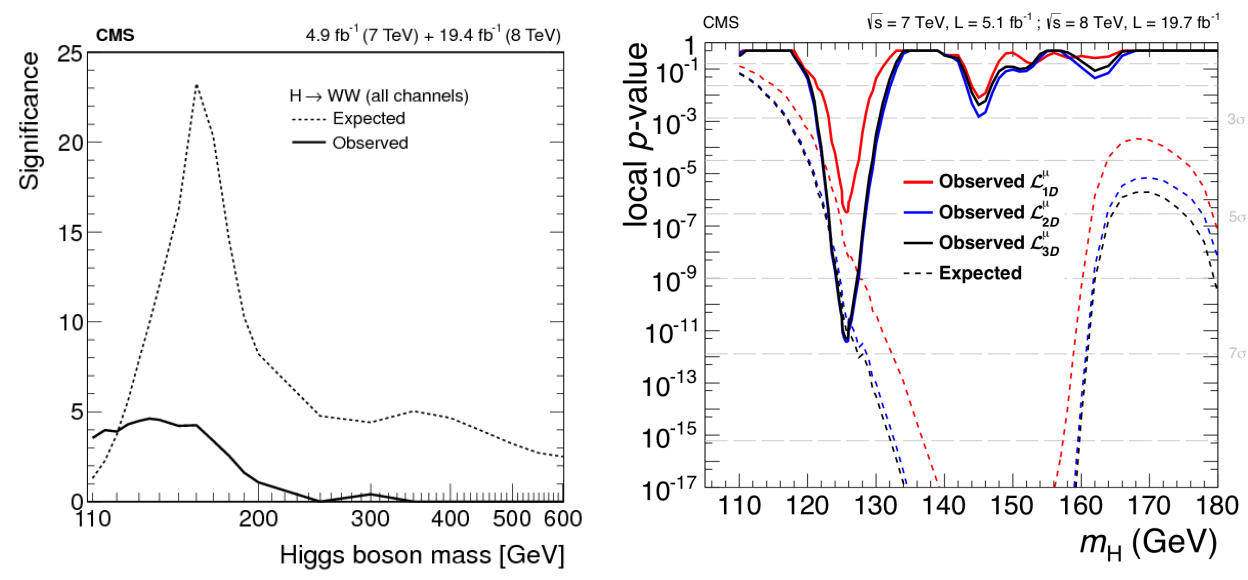

Fig. 6. Expected and observed significance as a function of the SM Higgs boson mass for the combination of all $H$ to $W W$ categories (left). Local $p$-value calculated for the $H \rightarrow Z Z$ analysis using $4 l$ mass (1D), $4 l$ mass and MELA (2D) and $4 l$, MELA, and $p_{\mathrm{T}}^{4 l}(3 \mathrm{D})$ signal discriminant (right).

The main search channel for Higgs decays into the other massive gauge boson is $H \rightarrow Z Z \rightarrow 4 l$, where $l$ stands for electrons or muons, and each $Z$ is allowed to decay into any lepton flavor. In this analysis [14], a search for a narrow peak in the invariant mass of four leptons $\left(m_{4 l}\right)$, over a limited continuous background is made. Since the full branching ratio $\mathrm{BR}(H \rightarrow Z Z \rightarrow 4 l)$ is very small, and some of the leptons can have small $p_{\mathrm{T}}$ the crucial element of this analysis is efficient lepton reconstruction at as low as possible $p_{\mathrm{T}}$. The signal extraction is made with a $3 \mathrm{D}$ fit using $m_{4 l}$, matrix element angular analysis (MELA) and $p_{\mathrm{T}}^{4 l}$. A very strong signal was observed with statistical significance of $6.8 \sigma$, as can be seen in Fig. 6 (right). The Higgs boson mass was estimated in this channel with a very high precision giving $m_{H}=125.6 \pm 0.4$ (stat.) \pm 0.2 (syst.) $\mathrm{GeV}$.

The search for $Z \rightarrow \gamma \gamma$ [16] looks for a narrow peak in the two photon invariant mass over a huge continuous background. The key point of this analysis is diphoton mass resolution, and precise, data driven, background estimation. To ensure robustness of the results, the CMS Collaboration has two analysis flavors: a classic cut-based and potentially more sensitive MVA based one. The results of both analysis flavours are statistically consistent. Figure 7 shows the $95 \%$ C.L. limit as a function of $m_{H}$ for the MVA analysis, with a clear minimum around $m_{H}=125 \mathrm{GeV}$. The signal statistical significance corresponds to $3.2 \sigma$, while the best fit mass is equal to $m_{H}=125.4 \pm 0.5$ (stat.) \pm 0.6 (syst.) $\mathrm{GeV}$. 


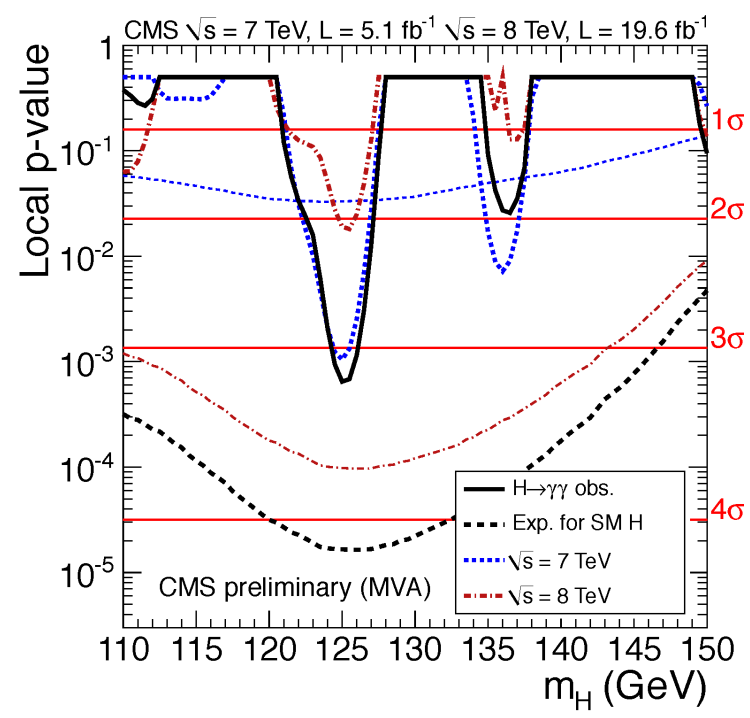

Fig. 7. Local $p$-value calculated for the $H \rightarrow \gamma \gamma$ analysis.

\section{Combined result on couplings}

The summary [17] of the signal strength modifier found for each Higgs boson decay mode is presented in Fig. 8 (left). Within (sometimes large) errors all the modifiers are consistent with 1.0, which is the SM expectation.
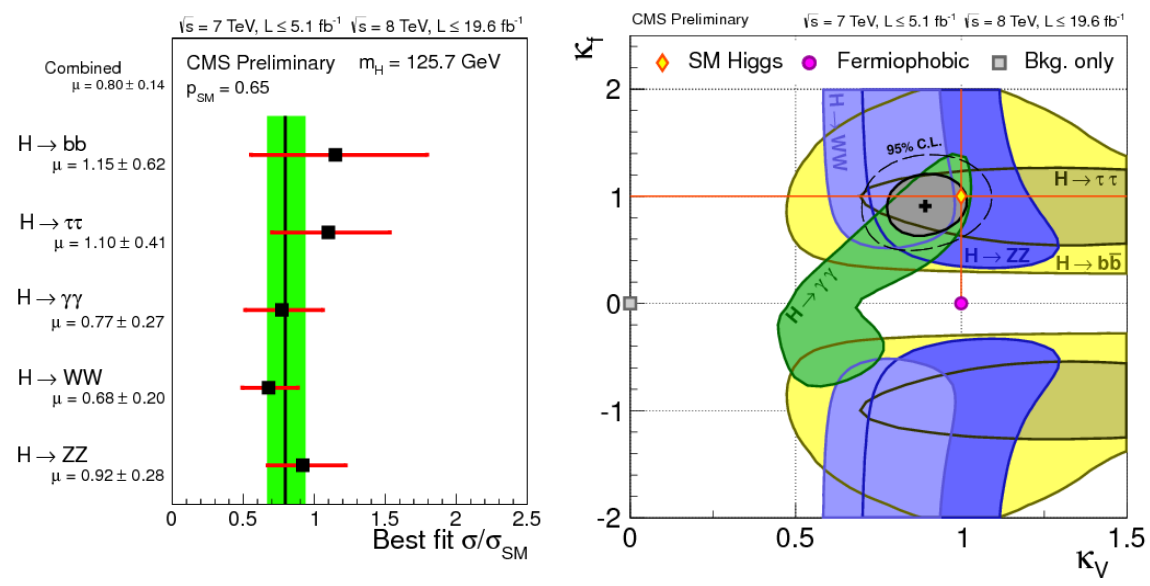

Fig. 8. Signal strength modifier for the combination (solid vertical line) and for subcombinations grouped by decay mode (left). Best fit and $1 \sigma$ contours for fermion vs. vector boson couplings in individual channels, and for the combination (right). 
The consistency with the SM can be studied also by analysis of the new particle couplings to fermions $\left(\kappa_{\mathrm{f}}\right)$ and vector bosons $\left(\kappa_{\mathrm{V}}\right)$. Since couplings affect analyses results both directly in the decay mode, and by a loop contribution to gluon-gluon fusion production mode, every analysis gives some contribution to both types of couplings. Figure 8 (right) presents summary of combined fit to $\kappa_{\mathrm{f}} v s$. $\kappa_{\mathrm{V}}$, and contributions from individual analyses. The results are fully compatible with the SM hypothesis.

\section{Spin and parity}

Full identification of the new particle as the Higgs boson requires estimation of its quantum numbers: spin $(\mathrm{S})$ parity $(\mathrm{P})$ and charge conjugation (C). Angular correlations between decay products momenta are often sensitive to spin and parity of the original particle. The $H \rightarrow W W, Z Z$ and $\gamma \gamma[14,15,18]$ decays have been analyzed to estimate the spin and parity of the Higgs boson. The $\gamma \gamma$ final state uses angle between the two photons calculated in the so-called Collins-Sopper frame [19]. The angular distribution for decay of a spin-0 is uniform in this frame, but the analysis selections significantly alter it as can be seen from Fig. 9 (left). The $H \rightarrow \gamma \gamma$ data are compatible with $0^{+}$hypothesis, but still the spin- 2 one cannot be excluded.
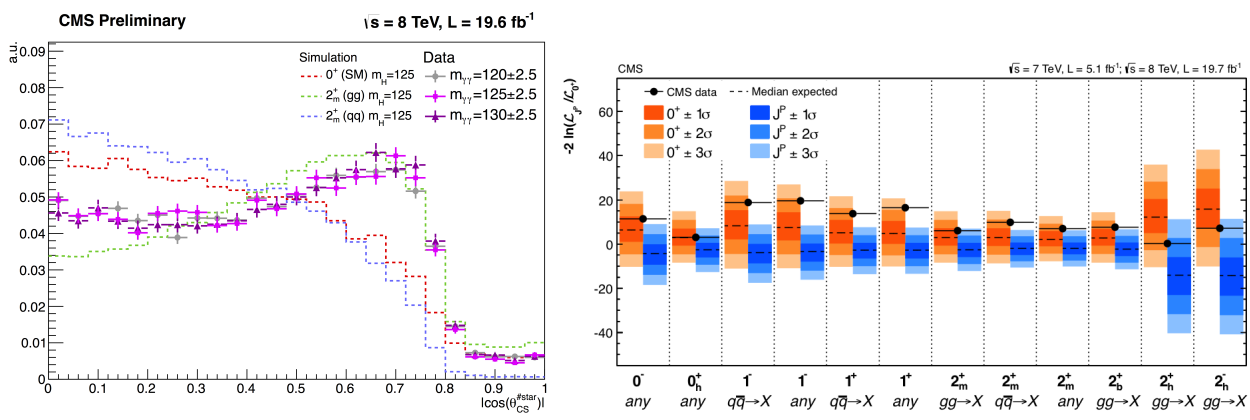

Fig. 9. The distribution of $\left|\cos \left(\theta^{*}\right)\right|$ after the selection cuts plotted with the data from three different $5 \mathrm{GeV}$ windows, and for three spin models in the $H \rightarrow \gamma \gamma$ analysis (left). The expected and observed values for the test-statistic distributions for the spin and parity hypotheses tested with respect to the SM Higgs boson in the $H \rightarrow Z Z \rightarrow 4 l$ analysis (right).

The $H \rightarrow Z Z \rightarrow 4 l$ analysis studied compatibility of data with number of spin and parity hypotheses showing that data is consistent with $0^{+}$hypothesis of SM. Figure 9 (right) presents summary of results for each hypothesis. The colored bands and shown $1 / 2 / 3 \sigma$ regions around the median expected value for the SM Higgs boson hypothesis (orange, left band) or alternative hypothesis (blue, right band), while the black point represents the observed value. 


\section{The MSSM results}

The Higgs sector in theories extending the SM are usually much richer than in the SM. The most popular one, Minimal Supersymmetric Standard Model (MSSM), predicts five physical Higgs bosons: three neutral: scalar $h$, $H$, and pseudoscalar $A$, and two charged $H^{ \pm}$. To date the most MSSM parameter space restricting results come from $H, h, A \rightarrow \tau \tau$ decay mode [20]. In this analysis, five from six possible $\tau \tau$ decays are analyzed: $\mu \tau_{\mathrm{h}}, e \tau_{\mathrm{h}} \tau_{\mathrm{h}} \tau_{\mathrm{h}}$, $e \mu, \mu \mu$. The analysis flow is similar to the one of SM, with an important difference on the event categorization. In the MSSM the two main production modes are gluon-gluon fusion, and associated production with two $b$-quarks. The latter is dominant for large ratio of vacuum expectation value of two Higgs doublets of MSSM, denoted as $\tan (\beta)$. To follow this pattern, the events are split into two categories: with and without a $b$-tagged jet. Since no signal was observed, a 95\% C.L. exclusion limit was calculated. Figure 10 (left) presents the exclusion region on the parameter space spanned by the pseudoscalar mass, $m_{A}$, and $\tan (\beta)$ calculated for one of benchmark MSSM scenarios [21].
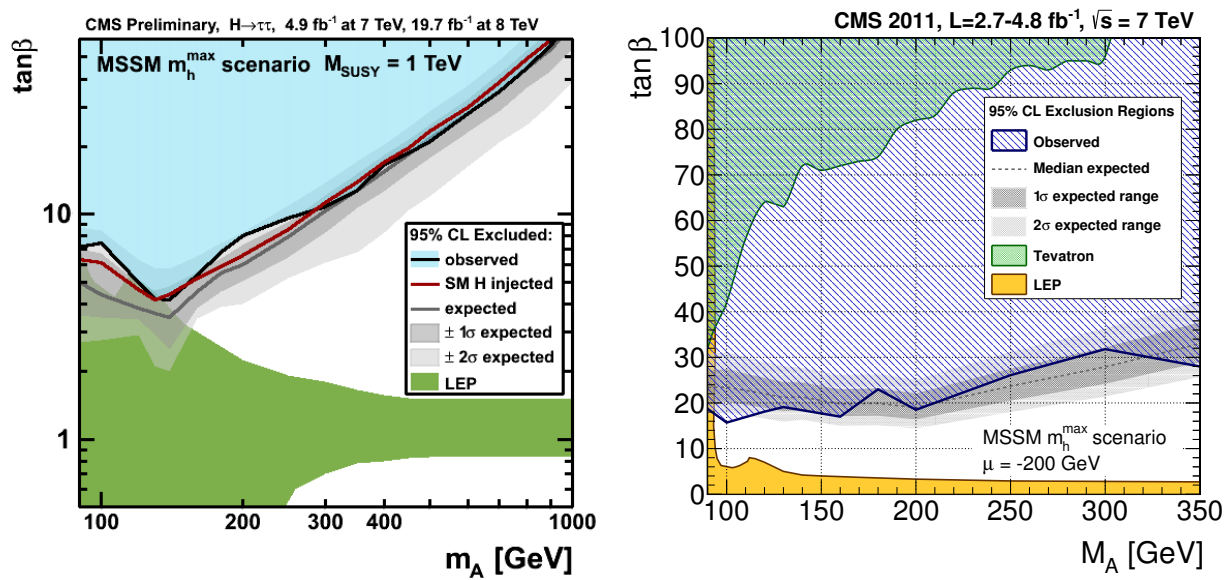

Fig. 10. Observed upper limits at $95 \%$ confidence level on $\tan (\beta)$ as a function of $M_{A}$ in the $\tau \tau$ (left), and $b \bar{b}$ (right) analyses calculated for the $m_{h}^{\max }$ benchmark scenario.

Complementary analysis looking at the $H \rightarrow b \bar{b}$ is one of few analyses looking at a fully hadronic final state. The full dataset of LHC Run 1 is still being processed, and only a result from first $4.8 \mathrm{fb}^{-1}$ has been published in [22]. Since no signal was observed, a 95\% C.L. exclusion limit was calculated. Figure 10 (right) presents the exclusion region. 


\section{Conclusions}

The CMS Collaboration analyzed 5.1(19.6) $\mathrm{fb}^{1}$ of data collected at $\sqrt{s}=$ $7(8) \mathrm{TeV}$ in search for the SM Higgs boson using number of decay and production modes. A strong, $6.8 \sigma$, signal was observed in the $H \rightarrow Z Z \rightarrow 4 l$ analysis, with results in other decay modes fully compatible with the SM expectations. Spin and parity statistical analyses are compatible with $0^{+}$ hypothesis of SM Higgs boson, although spin admixture still cannot be excluded. Searches for additional Higgs bosons predicted by extensions of SM, in particular MSSM, did not found any signal yielding exclusion limits in large region of benchmark parameter space.

\section{REFERENCES}

[1] G. Aad et al. [ATLAS Collaboration], Phys. Lett. B716, 1 (2012) [arXiv:1207.7214 [hep-ex]].

[2] S. Chatrchyan et al. [CMS Collaboration], Phys. Lett. B716, 30 (2012) [arXiv:1207.7235 [hep-ex]].

[3] G. Aad et al. [ATLAS Collaboration], JINST 3, S08003 (2008).

[4] S. Chatrchyan et al. [CMS Collaboration], JINST 3, S08004 (2008).

[5] S. Heinemeyer, C. Mariotti, G. Passarino, R. Tanaka (Eds.) [LHC Higgs Cross Section Working Group], Handbook of LHC Higgs Cross Sections: 3. Higgs Properties, CERN-2013-004 (CERN, Geneva, 2013), [arXiv:1307.1347 [hep-ph]].

[6] S. Chatrchyan et al. [CMS Collaboration], arXiv:1401.5041 [hep-ex].

[7] S. Chatrchyan et al. [CMS Collaboration], CMS-PAS-HIG-13-007.

[8] S. Chatrchyan et al. [CMS Collaboration], Phys. Rev. D89, 012003 (2014) [arXiv:1310.3687 [hep-ex]].

[9] S. Chatrchyan et al. [CMS Collaboration], arXiv:1401.6527 [hep-ex].

[10] S. Chatrchyan et al. [CMS Collaboration], CMS-PAS-HIG-13-020.

[11] S. Chatrchyan et al. [CMS Collaboration], CMS-PAS-HIG-12-025.

[12] S. Chatrchyan et al. [CMS Collaboration], CMS-PAS-HIG-13-019.

[13] S. Chatrchyan et al. [CMS Collaboration], CMS-PAS-HIG-13-015.

[14] S. Chatrchyan et al. [CMS Collaboration], arXiv:1312.5353 [hep-ex].

[15] S. Chatrchyan et al. [CMS Collaboration], J. High Energy Phys. 1401, 096 (2014) [arXiv: 1312.1129 [hep-ex]].

[16] S. Chatrchyan et al. [CMS Collaboration], CMS-PAS-HIG-13-001.

[17] S. Chatrchyan et al. [CMS Collaboration], CMS-PAS-HIG-13-005.

[18] S. Chatrchyan et al. [CMS Collaboration], CMS-PAS-HIG-13-016.

[19] J.C. Collins, D.E. Soper, Phys. Rev. D16, 2219 (1977). 
[20] S. Chatrchyan et al. [CMS Collaboration], CMS-PAS-HIG-13-021.

[21] M.S. Carena, S. Heinemeyer, C.E.M. Wagner, G. Weiglein, Eur. Phys. J. C26, 601 (2003) [arXiv:hep-ph/0202167].

[22] S. Chatrchyan et al. [CMS Collaboration], Phys. Lett. B722, 207 (2013) [arXiv:1302.2892 [hep-ex]]. 